\title{
Deleterious CDK12 Gene Mutation
}

National Cancer Institute

\section{Source}

National Cancer Institute. Deleterious CDK12 Gene Mutation. NCI Thesaurus. Code C134505.

A change in the nucleotide sequence of the CDK12 gene that is associated with increased risk of disease. 